Eur. J. Clin. Chem. Clin. Biochem.

Vol. 31, 1993, pp. 115-119

(C) 1993 Walter de Gruyter \& Co. Berlin - New York

\title{
Abnormal Bilirubin Binding to Human Serum Albumin in a Patient with Unusual Myeloma Immunoglobulin G
}

\author{
By J.-M. Gulian ${ }^{1.2}$, Christiane Dalmasso ${ }^{1}$, Martine Pesquié ${ }^{2}, J .-R$. Harle ${ }^{3}$ and M. Charrel ${ }^{1.2}$ \\ 1 Laboratoire de Chimie Biologique, Faculté de Médecine, Marseille, France \\ 2 Service de Biochimie, Centre Hospitalier Universitaire, Timone, Marseille, France \\ ${ }^{3}$ Service de Clinique Médicale, Centre Hospitalier Universitaire, Timone, Marseille, France
}

(Received January 24/November 9, 1992)

Summary: The binding of bilirubin was analysed in the serum of a patient with unusual myeloma. This patient presented an acquired Von Willebrand's disease due to the presence of a ternary complex formed by one molecule of myeloma immunoglobulin G and two molecules of albumin (Harlé et al. La Presse Médicale 19, $1661-1664(1990))$.

The binding characteristics of bilirubin - number of sites $(n)$ and association constants $(k)$ - were estimated in the serum of the proband, and compared with those of purified human serum albumin and pooled normal human serum. The data suggest that complex formation between albumin and immunoglobulin induces abnormal bilirubin binding in the patient's serum, which becomes apparent when the bilirubin/albumin ratio exceeds 1.3. This abnormal binding may be due to conformational changes of the albumin molecule in the complex. The greatly increased number of lower affinity binding sites for bilirubin is certainly due to the abnormally high concentration of myeloma immunoglobulin $G$ in the serum of the patient.

\section{Introduction}

Human serum albumin is a macromolecule involved in binding and transport of low-molecular-mass compounds in blood plasma. The interaction of these endogenous or exogenous ligands with albumin depends on the number of binding sites and the values of the related association constants. Theoretical analyses of binding have been developed by several authors $(1-6)$; for independent classes of sites, equilibrium can be defined by the equation:

$$
r=\sum_{i=1}^{m} \frac{n_{i} k_{i} A}{1+k_{i} A}
$$

where

$r$ represents the moles of bound ligand per mol of macromolecule,

$\mathrm{m}$ the number of classes of binding sites,

$n_{i}$ the number of sites in class $i$, $\mathrm{k}_{\mathrm{i}}$ the average association constant for that class and A the concentration of unbound ligand.

Binding properties of albumin are dependent on the integrity of the regions of the macromolecule involved in the association process. Many studies of interactions between albumin and small molecules have been reported, and localizations of binding sites for several compounds have been published (see review (7)).

In this report, we study the binding properties of albumin in a patient with acquired Von Willebrand's disease. Using electrophoresis, we earlier showed that the serum of this patient does not contain free albumin, but a complex of myeloma immunoglobulin $G$ kappa with albumin; this complex consists of one molecule of immunoglobulin $G$ linked to two molecules of albumin by disulfide bridges (8). It seems likely that the covalent bonding between the two species affects the spatial structure of the molecules 


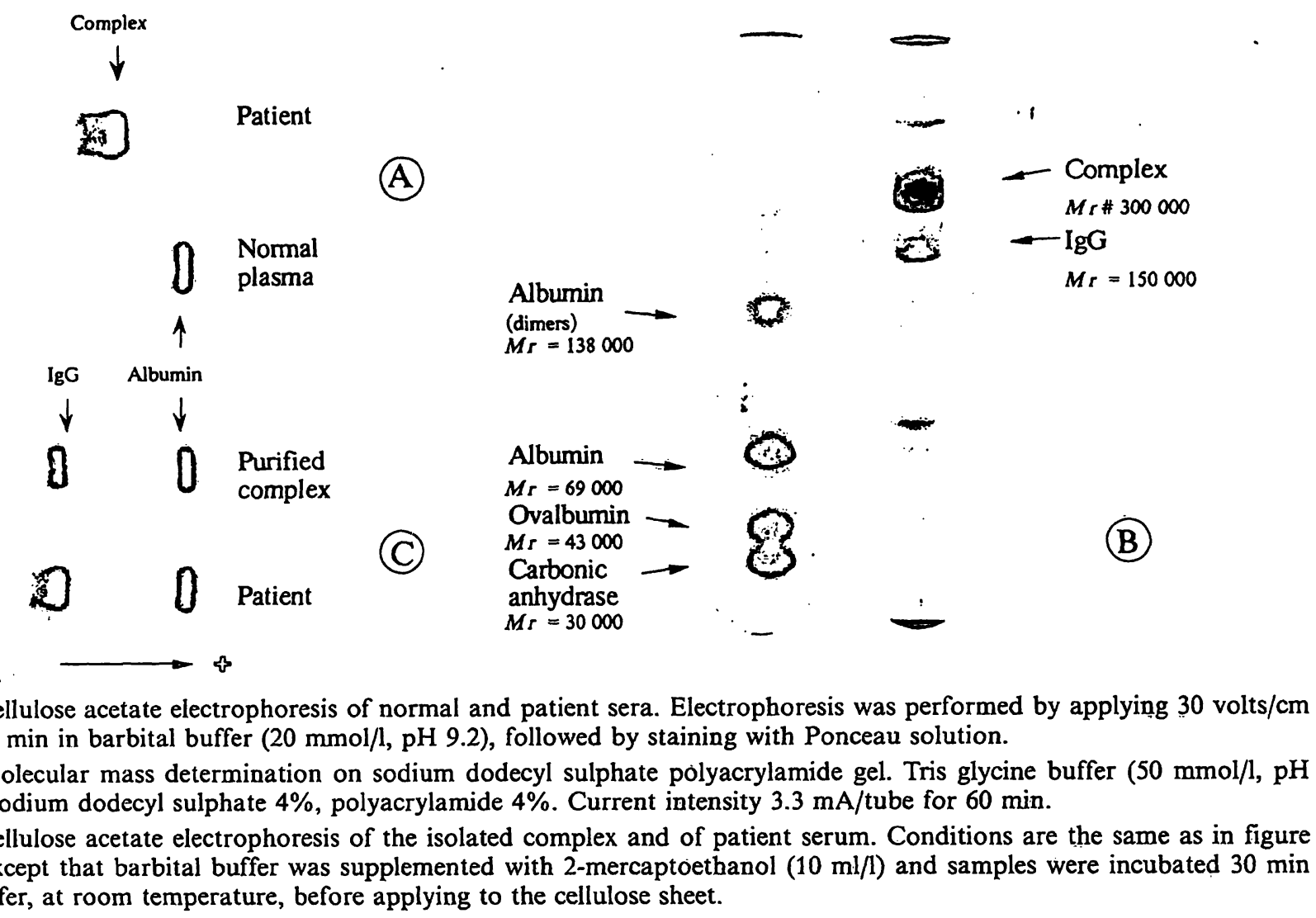

and consequently the binding of ligands to albumin. To investigate this possibility, we compared the binding equilibrium of bilirubin in the blood serum of this patient with the binding data for albumin. The nonbound bilirubin was determined by oxidation with hydrogen peroxide and horseradish peroxidase ${ }^{1}$ ), assuming that the oxidation rate was greater than the dissociation rate of the bilirubin-albumin complex $(9$, 10).

\section{Materials and Methods}

Albumin (3.3 mmol/l, Centre de Transfusion Sanguine de Montpellier) was diluted to a concentration of $500 \mu \mathrm{mol} / \mathrm{l}$ in 0.1 $\mathrm{mol} / \mathrm{l}$ phosphate buffer $\mathrm{pH}$ 7.4. Bilirubin was dissolved in isotonic saline containing $0.1 \mathrm{~mol} / \mathrm{l}$ sodium hydroxide, then added to the patient's serum or albumin solution. Total bilirubin was measured by the Jendrassik-Michaelson-Bruckner method on an Astra $^{\mathrm{TM}}$ analytical system; albumin concentration was determined by the Doumas method (11); free bilirubin by the method of Jacobsen \& Wennberg (10), using horseradish peroxidase (EC 1.11.1.7). Because of the low solubility of bilirubin, and the possibility of dimerization at neutral $\mathrm{pH}$ (12), or self-aggregation at high concentrations, we investigated molar total bilirubin/albumin ratios only up to a value of 2.5 .

In this molar ratio range, and with the known affinity of albumin for bilirubin, the molar ratio of bound bilirubin to albumin and the molar ratio of total bilirubin to albumin are very close. The intra-assay coefficients of variation are: $\pm 3 \%$ for lotal bilirubin, $\pm 2 \%$ for albumin and $\pm 4 \%$ for free bilirubin.

') Enzyme: horseradish peroxidase (EC 1.11.1.7)

\section{Case report}

A 63 year-old male was admitted for repeated nose-bleeding to the Centre Hospitalier de la Timone, Marseille, France. On admittance, total serum proteins were $120 \mathrm{~g} / \mathrm{l}$; albumin was 600 $\mu \mathrm{mol} / \mathrm{l}$; cellulose acetate electrophoresis revealed the absence of albumin and the presence of an abnormal fraction in the $\alpha_{2^{-}}$ globulin region (fig. 1A). An albumin-immunoglobulin $G$ complex was isolated on Sephacryl S-300 with a relative molecular mass of 300000 (fig. 1B); after reduction with 2-mercaptoethanol this complex dissociated into immunoglobulin $G$ and albumin (fig. 1C). After separation on a Sephadex G-100 column, we demonstrated that it was composed of one molecule of immunoglobulin $G\left(M_{r} 150000\right)$ and two molecules of albumin $\left(M_{\mathrm{r}} 69000\right)(8)$.

\section{Results}

Figure 2 shows that the molar ratio, total bilirubin/ albumin, plotted versus the values for free bilirubin was a curve for albumin and nearly a straight line for our patient's serum. For molar ratios approximately $\leq 1.3$, the two curves are close, indicating a similar partition of free and albumin-bound bilirubin, but above this value the concentration of free bilirubin increases more rapidly in the presence of purified albumin than in the presence of the patient's serum.

The binding values were calculated by using the following linear transformations;

$\frac{\text { free bilirubin }}{\text { molar ratio total bilirubin/albumin }}=f$ (free bilirubin) 
and the reciprocal plot

$$
\frac{1}{\text { free bilirubin }}=\mathrm{f}\left(\frac{1}{\text { molar ratio total bilirubin/albumin }}\right) \text {, }
$$

two graphical representations giving a better estimate for intercepts on the coordinate axes (6). The equations of curves are determined by computerized second-order non-linear least-square curve fitting and the intercepts are calculated. Based on the hypothesis of two independent classes of binding sites, and using the equations developed by Klotz \& Hunston (4), we determined the presence of two sites, with the association constants: $\mathrm{k}_{1}=3.8 \cdot 10^{7} \mathrm{l} / \mathrm{mol}$ and $\mathrm{k}_{2}=1.2$ $\cdot 10^{7} \mathrm{l} / \mathrm{mol}$ for albumin. These values are in good agreement with the results reported in previous work $(13-16)$. The computer-generated curve based on these values was in close agreement with the experimental plot (fig. 2).

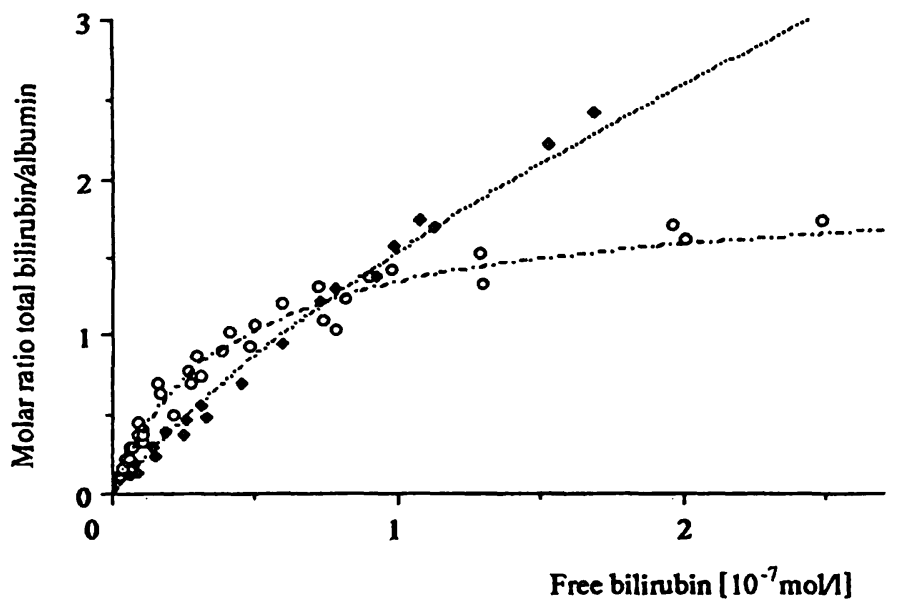

Fig. 2. Binding isotherms for bilirubin at $\mathrm{pH} 7.4$ and $25^{\circ} \mathrm{C}$. Experimental curves: $\mathrm{O}$ albumin; - our patient. Computer generated curves using calculated binding quantities: -....... simulation for albumin $\left(n_{1}=1, k_{1}=3.8\right.$ $\left.\cdot 10^{7} \mathrm{l} / \mathrm{mol}, \mathrm{n}_{2}=1, \mathrm{k}_{2}=1.2 \cdot 10^{7} \mathrm{l} / \mathrm{mol}\right) ; \cdots \cdots . .$. simulation for our patient $\left(n_{1}=1, k_{1}=1 \cdot 10^{7} \mathrm{l} / \mathrm{mol} ; n_{2}=17\right.$, $\mathrm{k}_{2}=6.4 \cdot 10^{5} \mathrm{l} / \mathrm{mol}$ )

It was assumed that the serum of our patient also manifested two non-interacting classes of binding sites. The total number of sites was obtained from the inverse of the ordinate intercept of the reciprocal plot. This value, 13.8, was rounded off to the nearest integer, 14. The number of classes and association constants were calculated with the same method as for albumin. For the first class of sites, $\mathrm{k}_{1}=1.8 \cdot 10^{7}$ $1 / \mathrm{mol}$ and the number of sites in this class was $n_{1}=1$; the average value of the association constant for the 13 remaining sites was $k_{2}=6.4 \cdot 10^{5} \mathrm{l} / \mathrm{mol}$. The curve generated by computer, using these values, was close to the experimental plot, but an even better graphical fit was obtained with $\mathrm{k}_{1}=1 \cdot 10^{7} \mathrm{l} / \mathrm{mol}$, and 17 as the number of second-class sites (fig. 2).

\section{Discussion}

Albumin is a monomeric protein whose complete amino acid sequence (585 residues) is known (17). The molecule possesses 17 disulphide bridges, a single cysteine residue in position 34 and a single tryptophanyl residue in position 214. Brown et al. (18), on the basis of a high degree of homology between bovine and human albumin, proposed that human albumin was formed by three similar globular domains I, II and III. Each domain can be subdivided into two subdomains (A and B) mainly structured as $\alpha$-helices (19). From hydrodynamic measurements performed on bovine albumin, the molecule is depicted as an oblate ellipsoid with axes $14 \mathrm{~nm}$ by $4 \mathrm{~nm}$ (20). Recent studies of the three-dimensional structure of albumin (21) reveal that domains are connected by non-helical segments, residues 177 to 200 between domains I and II, and residues 369 to 392 between domains II and III. For this reason, despite the high percentage of amino acid residues involved in secondary structures (48\% $\alpha$-helix and $15 \% \quad \beta$-sheet (19)), albumin remains a flexible molecule which undergoes reversible conformational changes, particularly as a result of ligandbinding; certain conformational changes exert cooperative or antagonist effects on ligand-binding (see review (7)).

Albumin can bind one molecule of bilirubin with a very high affinity and a second molecule with a lower affinity. Jacobsen (22) suggests that residues 127297 are involved in the first site, and that lysine residue 240 may be linked to one of the carboxylate groups of bilirubin. At the same time, Berde et al. (23) reported that the first site may be between domains I and II. Brodersen et al. (24) have calculated, by fluorescence quenching, that the first bound bilirubin molecule is located $2.2 \mathrm{~nm}$ from the tryptophan residue; this distance is comparable with the value of $2.7 \mathrm{~nm}$ reported by Berde et al. (23). For the second fixation-site, the results are conflicting. The observations of Gitzelmann et al. (25) suggest that the second molecule of bilirubin is located in the region of amino acids $446-547$ (domain III) at $2.4 \mathrm{~nm}$ from tryptophan 214. On the other hand, Brodersen et al. (24) established that the second molecule of bilirubin is closely bound with the first, at a distance of $1.8 \mathrm{~nm}$ from tryptophan.

The different association constant and different number of binding sites for human serum albumin and the serum of our patient clearly indicate that complex 
formation between albumin and immunoglobulin modifies the binding isotherms of bilirubin (fig. 2). The fact that all the albumin is linked (fig. 1A) and that its primary structure is normal (indeed, the complex with myeloma immunoglobulin appears in vivo after plasmapheresis, as well as in vitro with normal human albumin) indicates that the differences observed reflect the resulting conformational changes of bound albumin. The serum of our patient contains one binding site with an affinity $\left(\mathrm{k}_{1}=1 \cdot 10^{7}-1.8\right.$ $\cdot 10^{7} \mathrm{l} / \mathrm{mol}$ ) intermediate between the two values determined for albumin $\left(\mathrm{k}_{1}=3.8 \cdot 10^{7} \mathrm{l} / \mathrm{mol}, \mathrm{k}_{2}=1.2\right.$ $\cdot 10^{7} \mathrm{l} / \mathrm{mol}$ ); our results also indicate that the secondary site is no longer operative, and that 13 to 17 sites with 30 -fold weaker affinity $\left(\mathrm{k}_{2}=6.4 \cdot 10^{5} \mathrm{l} / \mathrm{mol}\right)$ are present. The linkage of albumin to immunoglobulin by an S-S bond, probably derived from the sulphydryl of cysteine 34 whose reactivity is distinctive at this $\mathrm{pH}(20)$, can be expected to induce perturbations in the intramolecular interactions of domain $\mathrm{I}$, where this cysteinyl residue is located. Although domain I does not contribute to the binding mechanism of bilirubin, our data suggest that one of the two highaffinity sites is dramatically affected by the coupling with immunoglobulin G.

\section{References}

1. Klotz, I. M. (1946) The application of the law of mass action to binding by proteins: interactions with calcium. Arch. Biochem. 9, 109-117.

2. Scatchard, G. (1949) Attraction of proteins for small molecules and ions. Ann. NY. Acad. Sci. 51, 660-666.

3. Fletcher, J. E., Spector, A. A. \& Ashbrook, J. D. (1970) Analysis of macromolecule-ligand binding by determination of stepwise equilibrium constants. Biochemistry 9, $4580-4587$.

4. Klotz, I. M. \& Hunston, D. L. (1971) Properties of graphical representation of multiple classes of binding sites. Biochemistry 10, 3065-3069.

5. Klotz, I. M. \& Hunston, D. L. (1979) Protein affinity for small molecules: conceptions and misconceptions. Arch. Biochem. Biophys. 193, 314-328.

6. Thakur, A. K., Jaffe, M. L. \& Rodbard, D. (1980) Graphical analysis of ligand-binding systems: evaluation by Monte Carlo studies. Anal. Biochem. 107, 279-295.

7. Kragh-Hansen, U. (1981) Molecular aspects of ligand binding to serum albumin. Pharm. Rev. 33, 17-53.

8. Harlé, J. R., Lefevre, P., Pesquié, M., Aillaud, M. F., Dalmasso, C., Boucraut, J., Gulian, J. M., Karsenti, J. M., Bernard, D. \& Charrel, M. (1990) Maladie de Willebrand acquise, cryoglobulinémie et pseudo-analbunémie au cours d'une dysglobulinémie monoclonale. Presse Méd. 19, $1661-1664$.

9. Jacobsen, J. (1969) Binding of bilirubin to human serum albumin - Determination of the dissociation constants. FEBS Lett. $5,112-114$.

10. Jacobsen, J. \& Wennberg, R. P. (1974) Determination of unbound bilirubin in the serum of newborns. Clin. Chem. 20,783-789.
Bilirubin can interact with other sites in serum such as $\alpha_{1}$ - and $\alpha_{2}$-globulins $(26,27)$, but with one hundred to one thousand-fold less binding strength than the high-affinity site of albumin (27). It is clear that the existence of 14 to 18 total binding sites, determined in the serum of our patient, should not be attributed solely to albumin but also to other plasma proteins. Since the results obtained from healthy subjects do not display such differences compared with pure albumin solutions (data not shown), the results observed are certainly due to the high concentration of immunoglobulin G. In order to understand the perturbations induced, firstly by the association of albumin with immunoglobulin $G$, and secondly, by the abnormally high concentration of a protein other than albumin, we plan to study the binding characteristics of other ligands in the serum of our patient and of other patients with hypergammaglobulinaemia.

\section{Acknowledgements}

The authors wish thank A. McKenna for linguistic corrections and Mrs. V. Azria for typing this manuscript.

11. Doumas, B. T., Watson, W. A. \& Biggs, H. G. (1971) Albumin standards and the measurement of serum albumin with bromcresol green. Clin. Chim. Acta 31, 87-96.

12. Brodersen, R. (1966) Dimerisation of bilirubin anion in aqueous solution. Acta Chem. Scand. 20, 2895-2896.

13. Brodersen, R. (1979) Bilirubin: solubility and interaction with albumin and phospholipid. J. Biol. Chem. 254, 23642369.

14. Jacobsen, J. (1969) Binding of bilirubin to human serum albumin. FEBS Lett. 5, 112-114.

15. Knudsen, A., Pedersen, A. O. \& Brodersen, R. (1986) Spectroscopic properties of bilirubin-human serum albumin complexes: a stochiometric analysis. Arch. Biochem. Biophys. $244,273-284$.

16. Nelson, T., Jacobsen, J. \& Wennberg, R. P. (1974) Effect of $\mathrm{pH}$ on the interaction of bilirubin with albumin and tissue culture cells. Pediat. Res. 8, 963-967.

17. Dugaiczyk, A., Law, S. W. \& Dennison, O. E. (1982) Nucleotide sequence and the encoded amino acids of human serum albumin mRNA. Proc. Natl. Acad. Sci. USA 79, $71-75$.

18. Brown, J. R. (1976) Structural origins of mammalian albumin. Fed. Proc. Fed. Am. Soc. Exp. Biol. 35, $2141-$ 2144.

19. Sjöholm, I. \& Ljungstedt, I. (1973) Studies on the tryptophan and drug-binding properties of human serum albumin fragments by affinity chromatography and circular dichroism measurements. J. Biol. Chem. 248, 8434-8441.

20. Peters, T. (1975) The Plasma Proteins. pp. 133-181, Putnam FW, Academic Press, New York. 
21. Carter, D., He, X-M., Munson, S. H., Twigg, P. D., Gernert, K. M., Broom, M. B. \& Miller, T. Y. (1989) Three-dimensional structure of human serum albumin. Science 244, 1195- 1198.

22. Jacobsen, C. (1978) Lysine residue 240 of human serum albumin is involved in high-affinity binding of bilirubin. Biochem. J. 171, 453-459.

23. Berde, C. B., Hudson, B. S., Simoni, R. D. \& Sklar, L. A. (1979) Human serum albumin. Spectrosoopic studies of binding and proximity relationships for fatty acids and bilirubin. J. Biol. Chem. 254, $391-400$.

24. Brodersen, R., Knudsen, A. \& Pedersen, A. O. (1987) Cobinding of bilirubin and sulfonamide and of two bilirubin molecules to human serum albumin: A site model. Arch. Biochem. Biophys. 252, 561-569.
25. Gitzelmann-Cumarasamy, N., Kuenzle, C. C. \& Wilson, K. J. (1976) Mapping of the primary bilirubin-binding site of human serum albumin. Experientia 32, 768.

26. Hierowski, M. \& Brodersen, R. (1974) Covalent binding of bilirubin to agarose and use of the product for affinity chromatography of serum albumin. Biochim. Biophys. Acta $354,121-129$.

27. Lamola, A. A., Eisinger, J., Blumberg, W. E., Patel, S. C. \& Flores, J. (1979) Fluorometric study of the partition of bilirubin among blood components: basis for rapid microassays of bilirubin and bilirubin binding capacity in whole blood. Anal. Biochem. 100, 25-42.

Dr. J-M. Gulian

Laboratoire de Chimie Biologique

Faculté de Médecine

27 Bd Jean Moulin

F-13385 Marseille, Cedex 5

France 
.

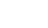

